\title{
Stand-Alone Wind Power System Operating with a Specific Storage Structure
}

\author{
M. Druga ${ }^{1}$, C. Nichita ${ }^{1}$, G. Barakat ${ }^{1}$ and E. Ceanga ${ }^{2}$ \\ ${ }^{1}$ Department of Electrical Engineering \\ G.R.E.A.H., Le Havre University \\ Le Havre Cedex, 76058 (France) \\ Phone number: +33 232 744336, Fax number: +33 232744348 \\ E-mail: Mirela.Druga@univ-lehavre.fr, Nichita@univ-lehavre.fr \\ ${ }^{2}$ Department of Electrical Energy Conversion System \\ "Dunarea de Jos" University of Galati \\ Galati, 800201 (Romania) \\ Phone/Fax number: +40 236 460754, e-mail: Emil.Ceanga@ugal.ro
}

\begin{abstract}
In this paper we analyze a low power wind system operating in autonomous mode. We present a control structure that ensures the maximization of wind energy conversion and the balance between required and produced power. We have studied two stand alone wind system structures based on the capacitor voltage control and the buffer battery operation. The simulation results have been obtained in Matlab/Simulink environment, using PowerSim toolbox.
\end{abstract}

\section{Key words}

Stand-alone wind system, control, storage energy, local power network, wind energy conversion.

\section{Introduction}

Energy is the support of all societal development, more than that, it plays a central role in the industrialised societies and developing countries. This is one of the reasons why clean, sustainable, environmentally and alternative energy resources are currently developed, mostly wind energy. After 1980s, the cost of electricity provided by wind energy has been drastically dropping. These cost reductions are due to new technologies, more efficient and more reliable wind turbines[1-4].

Nowadays, electrical energy savings at residential level, the production closer to places of habitation, have become a topic of lively debate for the energy demand satisfaction with a reduced price, economic and environmental $[5,6]$. Thus, the "small wind" applications became more and more popular in the isolated sites, these last years. Indeed, wind turbines usage is proved to be competitive regarding the traditional electrical energy sources, i.e. a diesel generator group. Therefore, study of the wind energy like a principal energy source for isolated sites, at a competitive price, has become a promising research way [7-9].
The present paper examines specific control methods related to a low power autonomous wind system conceived to run on an isolated site. Firstly, we present the operation modes according to the wind power level of the analysed stand-alone wind system. We treat the principles of the load regularization and the control devices which ensure the optimal mode of wind energy conversion, as well as the parameters of the electric power provided to the network. After that, the aspects concerning the system modelling and the system analyse are covered. Starting from the principles and the structural elements presented in section 2, we develop the stand-alone wind power system with a synchronous permanent magnet generator. Using Matlab/Simulink (SimPower) environment, simulations have been performed in order to analyse the operating behaviour of the considered system.

\section{The structure of a low power wind system}

This paper is focused on a low power wind system operating on an isolated site. The energy storage is realized with battery designed to operate in conjunction with a controlled load (see Fig. 1).

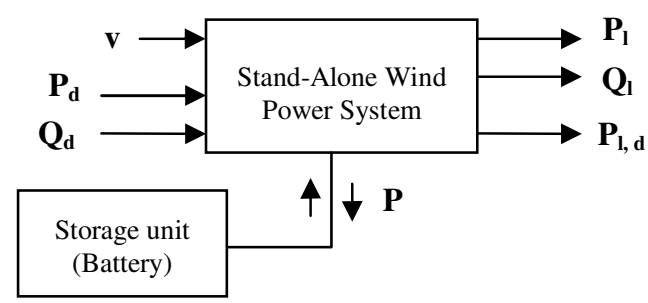

Fig. 1. Interaction of stand-alone wind power system with the local network 
As shown in Fig. 1, $v$ is the wind speed, $P_{d}$ and $Q_{d}$ are the powers corresponding to the consumption required in the local area network, $P_{l}$ and $Q_{l}$ are the real load consumptions. We make the hypothesis that the system has no classical supply i.e. a group diesel generator [10, 11]. When energy level is deficient, the stand-alone wind power system can not ensure the balance between produced powers and powers required by the load. Otherwise, when power developed by the wind generator exceeds the load consumption and the battery is fully refilled, the excess power will be dissipated on dissipation loads $\left(P_{l, d}\right)$.

The subsystems involved in the energy transfer which takes place from the turbine to the local network are illustrated synthetically in Fig. 2

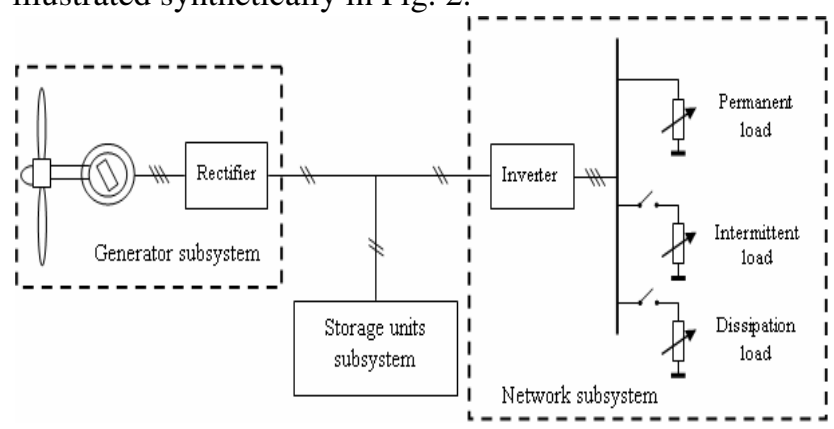

Fig. 2. Energy transfer subsystems

Three components of the stand alone wind system are presented in Fig. 2, which are: wind generator subsystem, local network subsystem and energy storage subsystem.

\section{A. Wind Generator subsystem}

The optimality of the whole system is defined in relation with the wind energy conversion maximization. This is achieved by maintaining the angular speed $(\Omega)$ at its optimal value using a cascade control loop, as shown in Fig. 3.

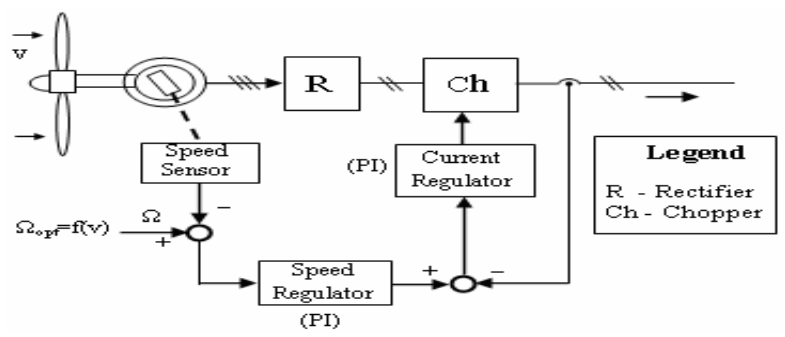

Fig. 3. Wind Generator subsystem structural schema

The chopper control being carried out by a current loop, the wind generator subsystem could be considered as a controlled current source.

\section{B. Local Network subsystem}

The local network load subsystem is composed by receivers that will be supplied in priority, by auxiliary loads handled by continuous or discontinuous commands (controlled loads) and by dissipation loads. It performs the load control using specific network switches (states
ON-OFF). The local network is associated to a local control loop for the AC bus voltage control.

\section{Energy Storage subsystem}

Two solutions for the energy storage subsystem were studied: electrostatic energy accumulation realized with a super capacitor storage and electrochemical energy accumulation (batteries). The last solution presents two operating alternatives: buffer battery or capacitor voltage control. Capacitor is the sensor that detects the existence of imbalance by voltage terminals variations. Offsetting this imbalance can be done in three ways: bidirectional energy transfer to the battery, intermittent loads control or both.

\section{Modeling methodologies}

The mechanical power developed by a wind-turbine can be expressed as:

$$
P=\frac{1}{2} C_{P} \rho \pi R^{2} v^{3}
$$

where, $C_{P}$ is the power coefficient, $\rho$ the air density and $R$ the wind-turbine rotor radius.

The power coefficient is the ration of the mechanical power at the turbine shaft to the power available in the wind. It is usually given as a function of tip-speed-ratio $\lambda$ :

$$
\lambda=\frac{\Omega R}{v}
$$

where $\Omega$ is the angular speed.

For the current turbines, the power coefficient presents a maximum for a certain value of the tip-speed-ratio, named specific optimal speed [12]. In consequent, the optimal angular speed according to the wind speed will be:

$$
\Omega^{o p t}=\left(\lambda^{o p t} / R\right) \cdot v
$$

In order to extract peak power, the rotor must be held at its optimal speed ratio, given by relation (3).

According to the available wind energy resource and the energy level required by the load, the stand-alone system could operate in the following scenarios:

1) Wind energy level in excess. In this case the required power level is ensured $\left(P_{l}=P_{d} ; Q_{l}=Q_{d}\right)$. The battery is refilled and the excess power is transferred in the dissipation load.

2) High wind energy level. Power level is ensured $\left(P_{l}=P_{d} ; Q_{l}=Q_{d}\right)$ and the rest of power is used for battery refill.

3) Average wind energy level. The power required by the load is provided jointly by two sources: aero generator and battery. Due to parallel operations, the battery can work in refill or discharge mode, according to the instantaneous value of the wind speed.

4) Reduced wind energy level. Although the aero generator and the battery are working in parallel in the local area network, the required power can not be furnished completely. The receivers connected in the 
local network must be treated on hierarchical basis, according to their priority. The adaptation of the total load of the local network to the energy resources available (aero generator and battery) is made by continuous or discrete commands of the receivers having a reduced priority level. This must take into account the discharge mode of the battery, which should not exceed an imposed limit.

5) Insufficient wind energy level. The wind speed is lower in comparison with the starting speed of the wind turbine. In this case, the battery ensures only the required power of the receivers having the highest level of priority.

We make the assumption that the battery will be able to ensure the energy request for priority receivers when the wind energy level is null, that it's indeed, a working hypothesis. In reality, it could happen that the battery cannot ensure the energetic needs of the priority receivers. In this situation, a security solution is employed, which consists of using a classical power group having adequate power to meet energy demand of the priority receivers.

Two solutions were mentioned previously, concerning the energy storage subsystem.

The first solution consists of using a super capacitor on the DC Bus. Capacitor voltage is sensitive to the imbalance between produced power and consumption. To achieve the balance between these two powers, a charge regulator is employed. The capacitor voltage is maintained constant by modifying the power of several receivers connected to the local network and operating as controlled loads, through continuous or discrete actions (switch ON/OFF). This solution is difficult to adopt (impractical solution), not only for the frequent changes of intermittent load's power regime, but especially for the fact that it is impossible to ensure the power continuity during periods with reduced wind energy level. Thereafter, this solution has not been the subject of a different simulation program because it can be analysed as a special case of the second solution.

In order to eliminate drawbacks of the first solution related to the continuity of energy supply during periods of wind deficit, a battery was introduced. However the wind potential should correspond to the needs of a standalone system and the battery choice should be done according to the site characteristics.

Firstly, the battery is associated to the capacitor voltage control structure. The simulation was made using the structural schema given in Fig. 4.

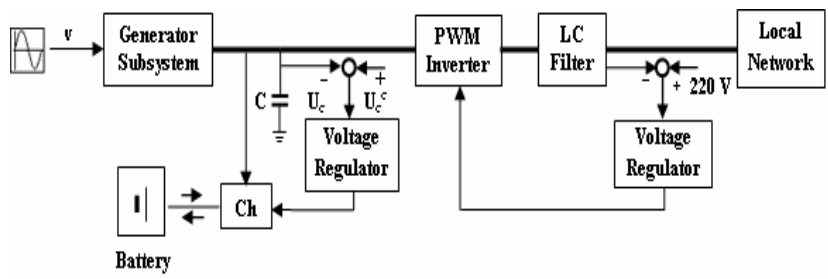

Fig. 4. Stand-alone wind system with capacitor and battery
The generator subsystem is considered as a controlled current source. It is necessary to underline that the current, generated by the wind source, is a random variable which has two components:

- A component which varies slowly, generated by the seasonal variation of the wind (on long intervals and average of time) and which determines the operating mode of the stand-alone wind system;

- A high frequency component, generated by the wind turbulences, which excites the control loops of the system, including the voltage control loop of network subsystem.

The energy storage subsystem (battery) is represented by a bidirectional current source. Controlling this source is function of the imbalance existing in the system, between the power debited by the wind turbine and the one consumed by the load. The energy disequilibrium is highlighted in this case also by the capacitor voltage changes. The connection with the local network is made through a PWM inverter, associated with a LC filter. The network load is composed by receivers that will be supplied in priority, by auxiliary loads handled by continuous or discrete commands and by dissipation loads. The two voltage regulators are PI (proportional integrator) controllers.

Secondly, the battery is working in the intermediary DC circuit (buffer battery). The structural schema is showed in Fig. 5.

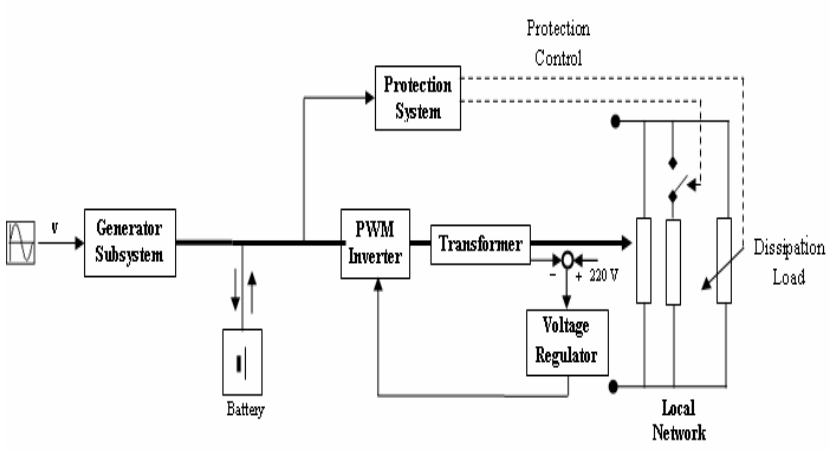

Fig. 5. Stand-alone wind system with buffer battery

This structure comprises some major modifications:

1) The battery voltage feeds the DC bus with a smaller voltage level $(96 \mathrm{~V}$ in the case of the analysed system);

2) For the same wind evolutions, the current loop of the generator subsystem must provide a more significant current level, to ensure the same power in the intermediate DC circuit;

3) The imbalances between the produced power and the consumed power determine, without any interventions, the charge/discharge battery operations. In this case, the capacitor becomes useless.

In the system diagram, we have eliminated the LC filter in order to check if the filtering effect of the transformer is sufficient to reduce the network harmonics. 
The DC bus voltage regulator is replaced by a protection system which operates in two situations:

- In the periods of wind energy deficit, when the battery voltage decreases below a threshold chosen between the nominal voltage and that of discharge, the auxiliary loads with a low priority level are disconnected.

- In the periods of exceed wind energy, when the battery voltage exceeds a threshold higher than nominal value, the dissipation load is connected.

\section{Simulation results}

Firstly, we present the simulation results when the energy storage subsystem is based on capacitor storage and battery energy accumulation.

The system is considered operating when the capacitor voltage is controlled by a PI command. This control loop is supposed to reduce the power imbalance in the studied system. The local power network is composed by receivers that must be supplied in priority, by auxiliary controlled loads and by dissipation loads.

The wind regime was supposed to be nominal, that is the instantaneous wind speed varies around the nominal value. In the case of considered wind, there is a surplus of produced energy, used for charging the battery.

In Fig. 6.A. the functioning diagram (local network coupling) of the controlled receivers, during 5 seconds, is showed. In Fig. 6.B. the RMS variations of the load current and the active power generated on the local network are presented.
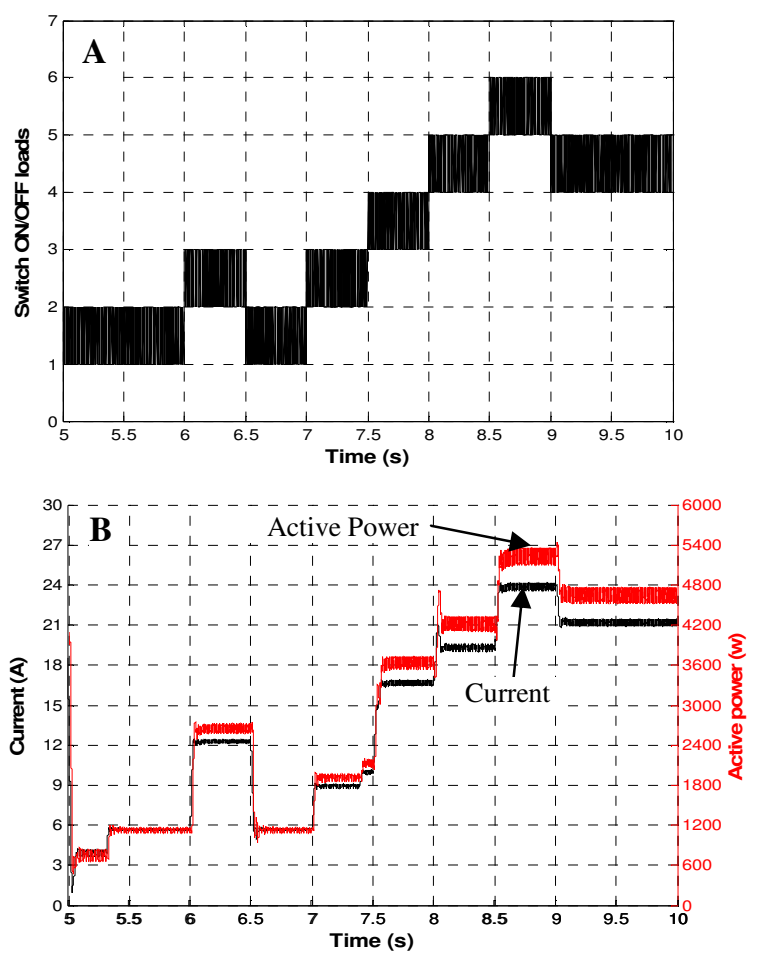

Fig. 6. Local network simulation results:

A) Local loads states (Connected/Disconnected).

B) Load current (black), Output active power (red).
The balance between the power produced by the wind turbine and that consumed for supplying the local network and charging the battery is realized by a PI control structure.

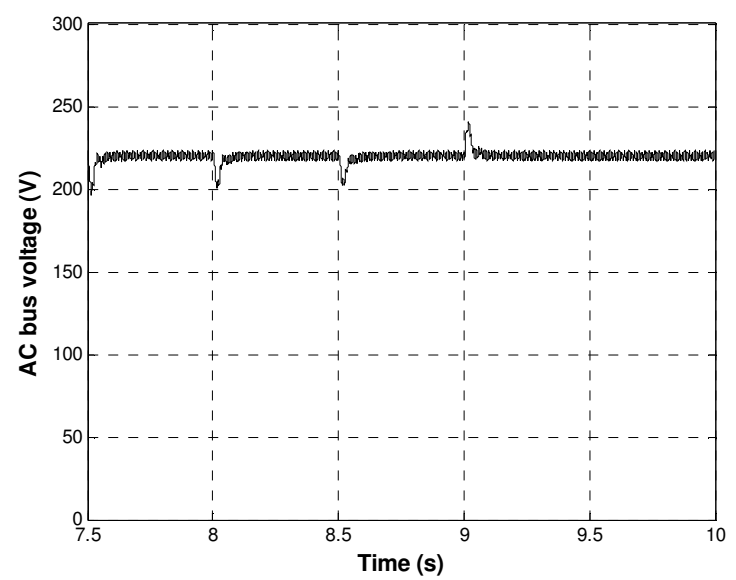

Fig. 7. AC bus voltage evolution

The AC bus voltage (network voltage) control functioning is given in Fig.7. We notice that the deviations around the effective value are weak, as long in amplitude, that in transitory regime duration. This behaviour was obtained in conditions of important load variations (see Fig.6.A), produced by the switching ON/OFF the receivers.

Secondly, the simulation results of the system operating with a buffer battery are exposed.

The simulation was carried out under conditions of significant variations of the load (the same load evolution diagram showed previously).

The transformer output voltage and output current can be examined over two periods $(40 \mathrm{~ms})$ in Fig. 8. We can notice the existence of high frequencies current components, but their influence is reduced thanks to the filtering effect of the transformer.

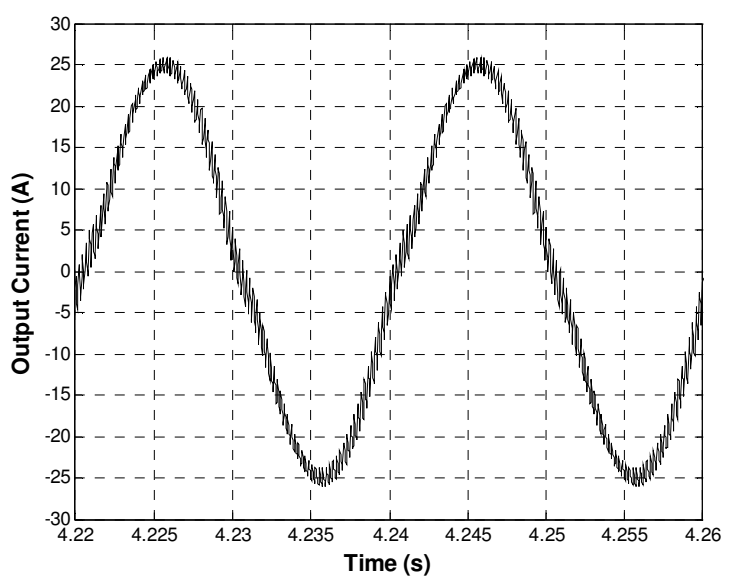

Fig. 8. Transformer output current evolution

The evolution of the AC bus voltage (local network voltage) during the starting up of the system is given in Fig. 9. According to this figure, it appears that the system 
provides a good balance between consumption and production of energy.

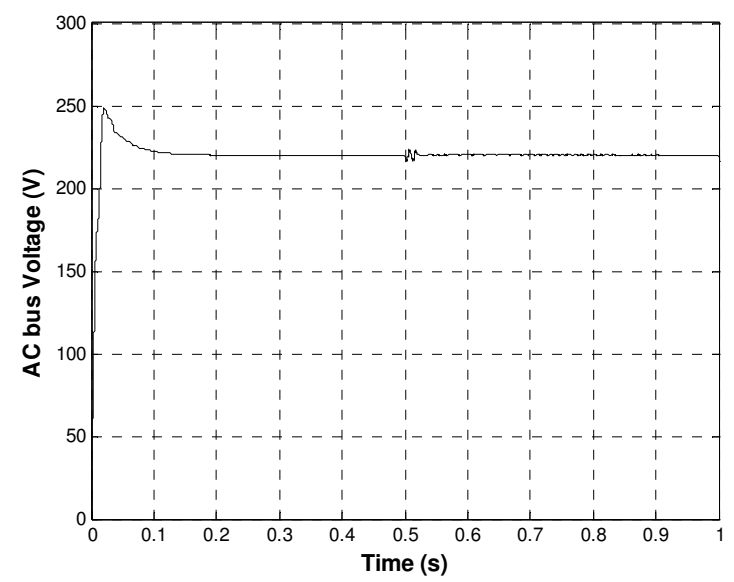

Fig. 9. AC bus voltage starting up evolution

This performance is achieved through a variable functioning of the battery, showed in Fig. 10, where the voltage level varies around the nominal value of $96 \mathrm{~V}$.

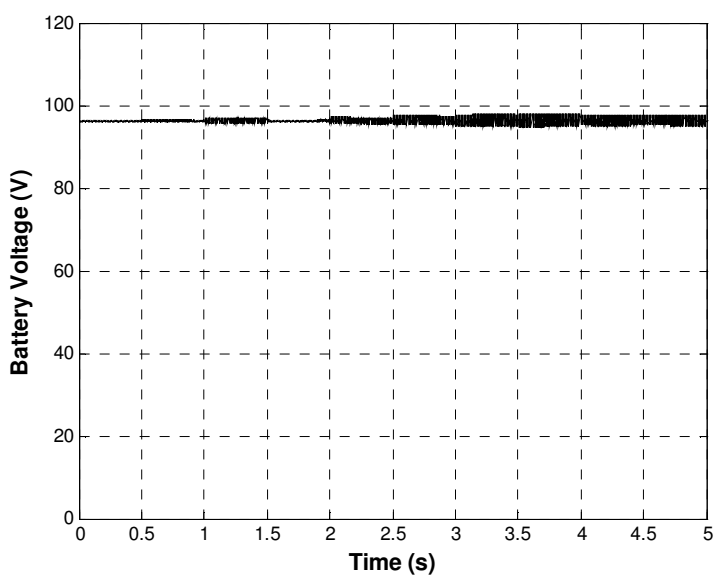

Fig. 10. Battery voltage evolution

The existence of a variable energy flow, from the aero generator side (because the wind speed variations), but also from the load side (produced by switching ON/OFF the receivers), generates fluctuations in the current charge/discharge of the buffer battery. These power fluctuations are reflected by small variations of the primary transformer AC voltage, showed in Fig. 11.

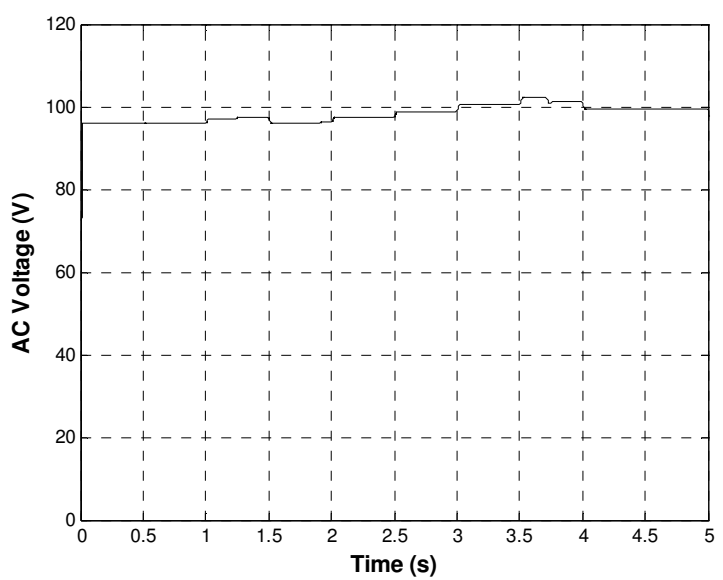

Fig. 11. Primary transformer AC voltage evolution
The primary AC voltage variations will be used by the protection system to capture the limit situations: connecting the dissipation load for an excess of power developed by the aero generator or disconnecting the local network system when there is wind deficit associated with battery exhaustion.

\section{Conclusion}

The solutions proposed for generator and network subsystems were analysed by digital simulation procedures. These solutions should perform the following basic functions, simultaneously:

1) Optimize the wind energy conversion;

2) Ensure the required parameters for the produced power;

3) Balance automatically between required and produced power, in conditions of energy accumulation.

The storage structure which uses the capacitor and the battery, allowed particularly that the system operates for a short time without battery. When the wind energy level is sufficient for the system consumption, maintenance battery operations can be made, while it is disconnected from the system. This solution is more expensive because a bi-directional chopper between the battery and the capacitor is used. Moreover it imposes higher requirements for the inverter that operates with a significant level of the DC bus voltage.

The energy storage subsystem with a buffer battery requires the permanent presence of the battery in the system. The advantages of this solution are the simplicity of the equipment and a reduced voltage level in the intermediary DC circuit. This voltage level reduces the over voltages of the PWM inverter, and consequently the protection systems will be less expensive to realize. We utilised for the latter solution a transformer that should be employed to connect the inverter to the network. The transformer performs the system's galvanic isolation over the network and satisfies also a filtering function of the high frequencies harmonics.

We intend for our future development to use the results of this work in order to minimize the global cost of the proposed Low Power Wind System designed to operate in stand alone applications.

\section{References}

[1] F. D. Bianchi, H. De Battista, and R. J. Mantz, Wind Turbine Control Systems: principles, modelling gain scheduling design (advances in industrial control), Lavoisier Ed. 2006.

[2] T. Ackermann and L. Soder, "An overview of wind energy-status 2002," Renewable and Sustainable Energy Reviews, vol. 6, 2002, pp. 67-127.

[3] A. D. Sahin, "Progress and recent trends in wind energy", Progress in Energy and Combustion Science, vol. 30, 2004, pp. 501-543. 
[4] G. M. Joselin Herbert, S. Iniyan, E. Sreevalsan, and S. Rajapandian, "A review of wind energy technologies", Renewable and Sustainable Energy Reviews, vol. 11, 2007, pp. 1117-1145.

[5] J. K. Kaldellis, "Optimum autonomous wind-power system sizing for remote consumers, using longterm wind speed data", in Applied Energy, vol. 71, 2002, pp. 215-233.

[6] R. Belfkira, C. Nichita, P. Reghem, and G. Barakat, "Modelling and optimal sizing of hybrid energy system", in Proc. of EPE-PEMC, Poland, 2008.

[7] N.E. Clausen, H. Bindner, S. Frandsen, J. C. Hansen, L. H. Hansen, and P. Lundsager, "Isolated systems with wind power - an implementation guideline", Riso National Laboratory, Roskilde, Denmark, 2001.

[8] J. Martinez, A. Morales, O. Probst, A. Llamas, and C. Rodreguez, "Analysis and simulation of a wind- electric battery charging system", International Journal of Energy Research, vol. 30, 2006, pp. 633646.

[9] M. Arifujjaman, M. T. Iqbal, and J. E. Quaicoe, "Energy capture by a small wind-energy conversion system", Applied Energy, vol. 85, 2008, pp. 41-51.

[10] K. C. Divya and J. Ostergaard, "Battery energy storage technology for power systems--An overview", in Electric Power Systems Research, vol. In Press, 2008, Corrected Proof.

[11] J. K. Kaldellis and D. Zafirakis, "Optimum energy storage techniques for the improvement of renewable energy sources-based electricity generation economic efficiency", in Energy, vol. 32, 2007, pp. 2295-2305.

[12] I. Munteanu, A. I. Bratcu, N.-A. Cutululis, and E. Ceanga, Optimal Control of Wind Energy Systems: Towards a Global Approach, Springer, 2008. 